\title{
Multi-scale Time Integration for Transient Conjugate Heat Transfer
}

\author{
L. He and M. Fadl \\ Department of Engineering Science, \\ University of Oxford, \\ Parks Road, \\ Oxford, OX1 3PJ, UK
}

\begin{abstract}
The quasi-steady assumption is commonly adopted in existing transient fluid-solid coupled convection-conduction (conjugate) heat transfer simulations, which may cause non-negligible errors in certain cases of practical interest. In the present work, we adopt a new multi-scale framework for the fluid domain formulated in a triple-timing form. The slow varying temporal gradient corresponding to the time scales in the solid domain has been effectively included in the fluid equations as a source term whilst short scale unsteadiness of the fluid domain is captured by a local time-integration at a given 'frozen' large scale time instant. For concept proof, validation and demonstration purposes, the proposed methodology has been implemented in a loosely coupled procedure in conjunction with a hybrid interfacing treatment for coupling efficiency and accuracy. The present results indicate that a much enhanced applicability can be achieved with relatively small modifications of existing transient conjugate heat transfer methods at little extra costs.
\end{abstract}

Keywords- Conjugate heat transfer; Transient fluid-solid coupling; Interface treatment; Unsteady flow; Multi-scale method.

\section{Introduction}

There is a wide range of engineering applications in which convective heat transfer plays a key role. Increasingly transient thermal characteristics during start-up, shut-down, load changing conditions of power generation operations (e.g. for steam turbines, gas turbine aeroengines) are of great interest. Conventional aerothermal designs tend to use the heat transfer coefficient (HTC) as the main boundary condition for the conduction (and thus temperature field) solution of the solid domain. The HTC based methods would work well if HTC is predominantly determined by flow, and not strongly influenced by the wall temperature, as commonly assumed. This can be questionable as pointed out by Moffat [1]. 
In some cases, when there is a large amount of heat transfer associated with a large temperature difference (e.g. for a typical gas-to-wall temperature ratio in high pressure turbines of gas turbines), the near-wall flow can experience significant density changes and thus rebalancing between density and velocity. The markedly changed local flow field can in turn lead to a markedly different HTC distribution, e.g. as observed for a high pressure turbine blading by Maffulli and He [2]. Although the dependence of the HTC on solid wall temperature may be accounted for by further iterations between a fluid solution (CFD) and solid conduction solution (FE/FV), the strong inter-dependence between the fluid domain solution and the solid one increasingly points to the need to develop and apply coupled convection-conduction (so called 'conjugate') heat-transfer approaches.

For coupled fluid-solid solutions, a closely relevant issue arises from the time scale disparity between fluid and solid parts. The ratio between the two time scales can be up to $10^{4}$ for gas turbine blades [3]. This scale disparity should not affect the steady CHT solution behaviour (computational efficiency and accuracy) in any way significant as the domain dependent time-stepping for common CHT solvers can be readily adopted. However, the issue becomes acute for time-dependent transient CHT problems. Examples of practical interest include the thermal load prediction for an engine cycle with a total time scale in hours on the one hand, whilst certain local ramping processes have a time scale in seconds on the other hand (e.g. [4], [5] ). Another example of the practical need for transient CHT solutions is the increasingly flexible operations of conventional steam turbine generators as required to provide top ups matching the emerging renewable power generations (e.g. [6] ). When shorter start-up and shut-down processes are pursued, transient temperature distributions in solid domains will be far from being quasi-steady, leading to potentially high transient thermal loads, which need to be predicted with adequate transient methods.

The disparity in time scales has presented a huge challenge to direct time accurate integration methods for fluid-solid systems. Fully coupled unsteady CHT simulations using the same discretization in space and time for both fluid and solid domains can be prohibitively expensive. They are also subject to strong influences of two different types of numerical errors. Firstly for high frequency flow disturbances, the small penetration depth on the solid side of the interface leads to high truncation errors in a common finite difference (FD) based interface treatment as shown in [7], manifested in a strong mesh dependence as shown in [8]. On the other hand, the time step taken needs to be small enough to resolve high frequency fluid disturbances, which tends to be practically too small to capture meaningful differences in temperature in the solid domain, during the solution 
process marching in time. This means that there tends to be large rounding error accumulation in the solid temperature prediction after time marching over a very large number of steps. The problem is difficult for URANS where the relative time-scales for the two domains can differ by $10^{4}$, and much worse for LES where smaller time steps by one or two orders of magnitude would be required.

Loosely coupled CHT methods, where the fluid and solid domains are solved by separate solvers and coupled together at an interface iteratively have been more commonly adopted. Although an unsteady flow solver can be used and the coupling between the two solvers can be conducted in time, such as [9], the direct coupling of this kind between the fluid and solid domains integrated in parallel in time tends to be very difficult. On the other hand, the apparent scale disparity between the fluid and solid provides a seemingly justifiable motivation for a quasi-steady transient CHT approach, i.e. coupling a steady flow solver quasi-steadily with an unsteady solid conduction solver.

The quasi-steady approach has been widely adopted recently with various flow fidelities (e.g. [4], [10], [11], [12] [13]). As far as the fluid-solid interfacing is concerned, considerable attention has been paid to the convergence behaviour. Although as shown by Giles [14], a solid temperature (Dirichlet) condition taken for the fluid domain and a heat flux (Neumann) condition for the solid domain should be stable, recent stability analyses suggest a mixed (Robin) condition gives a faster convergence (e.g. [11] [13]). It is worth noting however that the physical interfacing condition of the temperature and heat flux continuity may be more difficult to be fully realised in a Robin condition than in the direct Dirichlet-Neumann condition.

The quasi-steady CHT developments have indicated a scope for developing a more general framework applicable to a wide range of time scales. In particular, a solid domain can be subject to a fluid domain with multiple temporal flow disturbances, which themselves have markedly different time scales. It then becomes even less clear when we can treat the flow completely quasi-steadily.

In many transient CHT cases of practical interest, although the overall time scale of situation is long (e.g. in minutes or hours), some of the local ramping phases of the process may happen in relatively short time (e.g. a large variation of the flow conditions in a subsecond time). Thus the quasi-steady approach is likely to be in considerable errors locally. For unsteady CHT problems where a steady mean flow state can be clearly defined, the errors in the temporal terms will have a reduced impact on the steady/time-mean solution, 
given the alternating (+/-) nature of the unsteady disturbances. The examples of this kind include unsteady CHT solution of a turbine bladerow subject to periodic unsteady temperature and velocity distortions (inlet hot streak and swirl), e.g. [15] [16]), or LES solutions of turbine at a steady mean flow condition (e.g. [17]). However, for rapid rampups/downs in a largely monotonic phase of an otherwise slow engine operation cycle, neglect of the corresponding contribution to the temporal term may lead to non-negligible errors. The overall quality of the solution for a monotonic transient process can be strongly influenced by the history effect through accumulated errors at each time step, so that relatively small errors in balancing the unsteady flow equations locally and temporarily may matter more prominently in the resultant transient thermal load later in time.

These previous developments based on the quasi-steady assumption lead to the primary motivation of the present work. Here we adopt a multi-scale approach to transient conjugate heat transfer, chiefly aimed at addressing the issue of a quasi-steady fluid behaviour as assumed. A general framework is proposed to enable the transient conjugate heat transfer computations to be carried out for a wide range of time scales in both solid and fluid domains without needing the quasi-steady assumption for the fluid part. The presentation of the general methodology is followed by the several case studies by using the method as implemented in a commercial solver.

\section{Methodology}

\subsection{Baseline Fluid and Solid Models}

The baseline flow model is the three dimensional unsteady compressible Navier-Stokes equations in a short form:

$$
\frac{\partial U}{\partial t}+R(U)=0
$$

At a high Reynolds number, effects of turbulence fluctuations can be modelled, where the flow governing equations become the density weighted (Favre) time averaged equations (RANS for a steady flow or URANS for an unsteady flow without resolving turbulence eddies). Furthermore, given the increasing recognition of strong influences of turbulence (thus eddy resolved modelling. e.g. LES) on convective heat transfer [17] and [18], we will also need to consider the feasibility and applicability of a transient CHT methodology to turbulent eddy-resolved flow solvers. In this context, we include an option 
of using the scale-adaptive simulation (SAS) by Menter et al [19] for coupled transient CHT solutions, which is a type of eddy-resolved turbulence modelling towards LES.

In the solid domain, the temperature field is governed by the unsteady conduction equation.

$$
\frac{\partial T}{\partial t}+S(T)=0
$$

If the same solver is to be used for the both fluid and solid domains, the unsteady solid conduction equation is equivalent to the fluid energy equation with zero velocities, and can be solved directly by using the same numerical scheme as that for the fluid part.

As in many well established CFD solvers, we consider the use of a time-marching method for driving a solution to converge. We thus introduce a pseudo time. As a baseline, the direct integration for an unsteady CHT system can be written in a dual timing form [20] with the backward $2^{\text {nd }}$ order approximation of the physical time gradient, driven to converge at a given physical time step $\mathrm{M}$ by sub-iterations through time-marching in :

$$
\begin{aligned}
& \left(\frac{\partial \mathbf{U}}{\partial \tau}\right)_{M}+\frac{1.5 \mathbf{U}_{M}-2 \mathbf{U}_{M-1}+0.5 U_{M-2}}{\Delta t}+R\left(\mathbf{U}_{M}\right)=0 \\
& \left(\frac{\partial T}{\partial \tau}\right)_{M}++\frac{1.5 T_{M}-2 T_{M-1}+0.5 T_{M-2}}{\Delta t}+S\left(T_{M}\right)=0
\end{aligned}
$$

\subsection{Two-scale Fluid-Solid Coupled System}

The disparate time scales for a coupled fluid and solid thermal system can be depicted for a flow variable as shown in Fig.1. If a direct time-accurate integration of the fluid-solid coupled system is adopted, the time-step small enough to resolve the fluid disturbances (unsteady vortical flow structures and/or large scale turbulence eddies) will have to be used. This would be expensive and furthermore a very small time step size for the solid domain may affect the accuracy of the resultant temperature field solution by accumulated effects of rounding errors (a small difference between two large numbers). Therefore the coupled system should be integrated by using a much larger time step suitable for the temporal resolution for the solid part, as shown in Fig.1b. 


\subsubsection{Flow Variable and Time-scale Decomposition}

A flow decomposition is taken primarily in order to better deal with the fluid-solid time scale disparity. In addition, the decomposition is introduced to enable accommodating scale-resolved solvers for turbulence eddies and other self-excited flow instabilities (e.g. unsteady shear layers/vortex-shedding) by URANS or LES.

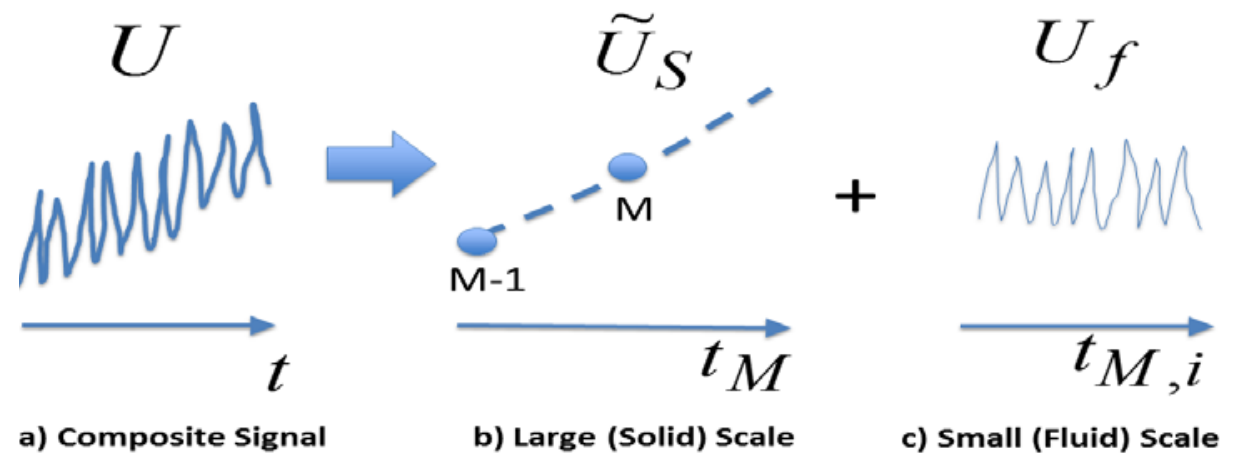

Fig.1, Timescale-dependent Flow Decomposition

A general flow variable can be expressed as

$U(x, t)=\tilde{U}(x, t)+U^{\prime}(x, t)$

$\tilde{U}$ is the flow variable after a low-pass filtering so that it only contains long wavelength disturbances comparable to the large time scales of the solid domain. More specifically, for any sampling point in a discrete time domain, the time instant $t$ is marked by a large scale time instant $\mathrm{t}_{\mathrm{M}}$ corresponding to a solid domain solution, and a small scale time instant $\mathrm{t}_{\mathrm{Mi}}$ relative to $t_{M}$.

$\mathrm{t}=\mathrm{t}_{\mathrm{M}}+\mathrm{t}_{\mathrm{Mi}}$

The filtering is effectively a time-averaging of small scale disturbances over a number of local time steps (denoted as 'fluid steps'),

$\tilde{U}\left(t_{M}\right)=\frac{1}{m} \sum_{i=1}^{m} U\left(t_{M i}\right)$

Where $\mathrm{m}$ is a sufficiently large number for a local time-averaging to be adequately obtained. Given the definition of time-filtering, it then follows, $\widetilde{U^{\prime}}=0$. 


\subsubsection{Two-scale Integrations of Flow Equations}

The unsteady flow equation after the low-pass filtering becomes:

$\frac{\partial \widetilde{U}}{\partial t}+\widetilde{R(U)}=0$

Note that $\widetilde{R(U)} \neq R(\widetilde{U})$ due to nonlinearity of convection terms in the flow equations.

In order to satisfy the above equation at a solid time instant $t_{M}$, noting that $\partial \widetilde{U^{\prime}} / \partial t=0$, we can rewrite the above equation as:

$\left(\frac{\partial \tilde{U}}{\partial t}\right)_{M}+\frac{1}{m} \sum_{i=1}^{m}\left[\frac{\partial U^{\prime}}{\partial t}+R(U)\right]_{i}=0$

Given that a filtered flow variable does not change with the local short time scale, for a given solid time instant $t_{M}$, the corresponding time-gradient of the filtered flow variable $\left(\frac{\partial \tilde{U}}{\partial t}\right)_{M}$ can thus be treated as a constant source term. As such, the above equation (Eq.8) will hold if the following equations hold at m local time steps respectively:

$\left(\frac{\partial \tilde{U}}{\partial t}\right)_{M}+\left[\frac{\partial U^{\prime}}{\partial t}+R(U)\right]_{i}=0 \quad$ (for $\left.\mathrm{i}=1,2, \ldots . \mathrm{m}\right)$

Similarly at this given solid time instant, the filtered part of the flow variable $\tilde{U}$ should be regarded as being frozen when integrating in the local fluid time. We thus have:

$\frac{\partial U^{\prime}}{\partial t}=\frac{\partial(U-\tilde{U})}{\partial t}=\frac{\partial U}{\partial t} \quad$ (for local time $\mathrm{t}=\mathrm{t}_{\mathrm{M} 1}, \mathrm{t}_{\mathrm{M} 2}, \ldots, \mathrm{t}_{\mathrm{Mm}}$ )

Based on the above reasoning, the time-domain unsteady flow equation to be solved locally at the given solid time step $\mathrm{M}$ becomes:

$\left[\frac{\partial U}{\partial t}+R(U)\right]_{i}=-\left(\frac{\partial \tilde{U}}{\partial t}\right)_{M} \quad$ (for local time step, $\mathrm{i}=1,2, . ., \mathrm{m}$ )

\subsubsection{Fluid-Solid Equations}

Using a time-marching solver by introducing a pseudo time $\tau$, we now have a triple-timing form of the unsteady flow equations.

$$
\frac{\partial U}{\partial \tau}+R(U)=-\left(\frac{\partial \tilde{U}}{\partial t}\right)_{M}-\left(\frac{\partial U}{\partial t}\right)_{m}
$$

where subscripts ' $M$ ' and ' $m$ ' denote the solid and fluid time scales respectively. In terms of the interactions between the two scales, the large scale physical time-gradient provides 
a base-value contribution to balancing the unsteady flow equations at each small time step, whilst the averaged small scale unsteady flow disturbances collectively change those of the large scales.

It is also useful to note that the small scale temporal resolution is introduced intentionally to resolve small scale self-excited unsteadiness (turbulence eddies, vortex shedding). For modelling consistence, we may consider an extreme case when the large scale time step is taken to be fine enough to resolve these self-excited unsteady disturbances directly. Then, the small scale time stepping does not provide any extra resolution, we thus should have, $(\partial \mathrm{U} / \partial \mathrm{t})_{\mathrm{m}} \rightarrow 0$, and the triple time stepping should reduce to the conventional dual time stepping.

For the solid domain on the other hand, the dual timing formulation remains as the large time step should be indeed determined by the required solid temporal resolution.

$\left(\frac{\partial \tilde{T}}{\partial \tau}\right)+S(\tilde{T})=-\left(\frac{\partial \tilde{T}}{\partial t}\right)_{M}$

For both domains, the temporal gradient terms in the physical time can be discretized in any chosen temporal integration scheme.

\subsection{Fluid-Solid Interface}

A general multi-scale framework for coupled fluid-solid conjugate heat transfer will be first presented, followed by three options for implementing a loosely coupled interfacing method.

\subsubsection{Multi-scale fluid-solid interface conditions}

\section{Energy Balance on Large Time Scale:}

At large solid time step $\mathrm{M}$, the locally time-averaged (filtered) heat flux on the fluid side must be the same as that on the solid side:

$\left(\widetilde{q_{f}}\right)_{M}=\left(\widetilde{q_{S}}\right)_{M}$

The discretized form (e.g. using one-sided FD) of large-scale flux balance can lead to the equation to obtain the large scale wall temperature $\widetilde{T_{w}}$ as in a standard steady CHT solution. As such, the physical condition of temperature and flux continuity across a fluidsolid interface is fully enforced for the filtered variables at each solid time step. 


\section{Energy Balance on Small Time Scale:}

For turbulence and coherent unsteady disturbances, we can assume that for a given spatial point, there exists a unique turbulence spectrum. The spectrum should determine the two key turbulence parameters: turbulence kinetic energy and length scale. This assumption is regarded justifiable and serves as the basis of the Fourier-spectrum based synthetic turbulence generation for LES inflow conditions [21]. It has also been used in the unsteady CHT solutions with LES for turbine blades by He [7].

In the context of the general framework, the low-pass filtering means that the high frequency unsteady disturbances have a zero time-mean when averaged over the local time scale. Therefore we can express them similarly in a Fourier-spectrum of $\mathrm{N}_{\mathrm{F}}$ harmonics, e.g. for fluid temperature fluctuations:

$$
T^{\prime}(x, t)=\sum_{k=1}^{N_{F}}\left(\hat{T}_{k} e^{i \omega_{k} t}+\hat{T}_{-k} e^{-i \omega_{k} t}\right)
$$

The harmonic balance for corresponding fluid and solid heat flux harmonics reads:

$\left(\hat{q}_{f}\right)_{k}=\left(\hat{q}_{s}\right)_{k}$

$$
\left(\mathrm{k}=1,2, \ldots . . \mathrm{N}_{\mathrm{F}}\right)
$$

which can be used to generate $\mathrm{N}_{\mathrm{F}}$ harmonic transfer functions to work out the corresponding wall temperature harmonics $\left(\widehat{T}_{f}\right)_{k}=\left(\widehat{T}_{s}\right)_{k} \quad\left(\mathrm{k}=1,2, \ldots . . \mathrm{N}_{\mathrm{F}}\right)$, as shown by He [7]. A key difference between the present framework and the previous one [7] is that the Fourier Transfer should be carried out in the local time $t_{M, i}(i=1,2, . ., n)$ for a given and fixed solid time instant $t_{\mathrm{M}}$. In this way, the physical conditions of temperature and heat flux across an interface can be enforced for all corresponding harmonic components.

It is pointed out the harmonics of wall temperature fluctuation as well as their impact on the large scale interface flux and temperature continuity tend to be small. The energy harmonic balancing of the small scale fluctuations are presented here for completeness of the general framework. They are not further examined in the test cases of the present paper.

\subsubsection{Solid Temperature and Fluid Heat Flux (Ts - $\mathbf{q}_{\mathrm{f}}$ ) Interface.}

A loosely coupled CHT solution is the one where fluid and solid domains are solved separately in sequence. Each consecutive pair of solutions for the two domains can be called a 'coupling cycle'. The interfacing conditions for a loosely coupled CHT are effectively the boundary conditions for each domain, updated once in each coupling cycle. 
The baseline boundary conditions for the loosely coupled CHT directly follow the common physical conditions of temperature and heat flux continuity across the interface as first articulated by Perelmans [22]. A stable interfacing treatment can be achieved by simply specifying a wall temperature condition for the fluid domain and specifying a heat flux for the solid domain iteratively as pointed out by Giles [14]. This simple Dirichlet (fluid) and Neumann (solid) interfacing treatment (denoted as 'Ts-qf') can be easily implemented as (marching from coupling cycle $\mathrm{N}$ to $\mathrm{N}+1$ ):

$\left(\widetilde{T_{f}}\right)_{N+1}=\left(\widetilde{T_{S}}\right)_{N}$

$\left(\widetilde{q_{S}}\right)_{N+1}=\left(\widetilde{q_{f}}\right)_{N+1}$

Clearly, when the coupling cycles converge, the above will be a direct manifestation of the physical temperature and flux continuity across the interface.

\subsubsection{HTC based Interface}

Newton's law of cooling as applied to the filtered variables should read:

$\tilde{q}=h\left(\widetilde{T_{f}}-\tilde{T} s\right)$

Based on the conventional wisdom, the heat transfer coefficient (HTC) h and the fluid driving temperature $\widetilde{T_{f}}$ are regarded as largely determined by the fluid mechanics with no (or only weak) dependence on wall temperature. Here, we intend to make use of this weak wall-temperature dependence locally. In coupling cycle $\mathrm{N}$, for a given solid wall temperature $\left(\widetilde{T_{S}}\right)_{N}$, we get a fluid solution and another one with a slightly different wall temperature $\left(\widetilde{T_{S}}\right)_{N^{\prime}}$ (e.g. by perturbing the wall temperature by $2-3 \%$ e.g. [2]). The corresponding heat fluxes from the two flow solutions $\left[\left(\tilde{q}_{f}\right)_{N},\left(\tilde{q}_{f}\right)_{N^{\prime}}\right]$ at the two wall temperatures $\left[\left(\widetilde{T_{S}}\right)_{N},\left(\widetilde{T_{S}}\right)_{N^{\prime}}\right]$ lead to a finite difference form for the HTC as a local invariant:

$$
h_{N}=\frac{\left(\widetilde{q_{f}}\right)_{N}-\left(\widetilde{q_{f}}\right)_{N}}{\left(\widetilde{T_{S}}\right)_{N}-\left(\widetilde{T_{S}}\right)_{N \prime}}
$$

The procedure of the two solutions for two unknowns also gives the value of the other local invariant $\left(\widetilde{T_{f}}\right)_{N}$. This two-point method is a consistent way of working out the HTC without an often somewhat arbitrarily defined fluid driving temperature. Both $h_{N}$ and $\left(\widetilde{T_{f}}\right)_{N}$ can then be used as the boundary condition for the solid domain, leading to $\left(\widetilde{T_{S}}\right)_{N+1}$ for the next coupling cycle. 


\subsubsection{Hybrid Interface Method}

It is generally observed that the HTC based interfacing treatment leads to a faster convergence than the direct sequential $\mathrm{T}_{\mathrm{s}}-\mathrm{q}_{\mathrm{f}}$ method (i.e. specifying temperature for fluid and flux for solid) [14]. However, a converged solution based on HTC does not necessarily lead to a full enforcement of the physical temperature and flux continuity at the interface. Note that the HTC based approach may be regarded as a kind of Robin type of boundary condition which has been extensively studied recently in the context of conjugate heat transfer coupling e.g. [11][13]. Once the HTC $\mathrm{h}$ and $\widetilde{T_{f}}$ are known and the solid thermal conductivity $\mathrm{k}$ is given, the boundary condition for the solid domain can be simply written as ( $\mathrm{y}_{\mathrm{n}}$ is the wall normal distance):

$k \frac{\partial \widetilde{T}_{S}}{\partial y_{n}}+h \widetilde{T}_{S}=h \widetilde{T}_{f}$

The above indeed shows the form of a Robin type condition for the solid temperature. Thus we can make an observation that although a Robin-type condition tends to give a faster convergence for a loosely coupled system, a more direct and fuller enforcement of the temperature and flux continuity would be preferred. Based on this consideration, we put forward a hybrid implementation here in the present work. The hybrid implementation is aimed at taking the advantages of the two options to enforce the physical condition whilst having an enhanced convergence of the coupling cycles. During a sequential coupling, we first use the HTC method to drive down temperature residuals quickly in initial cycles. Then we simply switch to the $T_{S}-q_{f}$ method to complete the remaining coupling cycles. As such, at the end of coupling cycles, a strong and direct enforcement of the physical T-q continuity at the interface can be realised. Typically 1-2 HTC cycles followed by 1-2 Ts $\mathrm{q}_{\mathrm{f}}$ cycles are found to be sufficient.

\section{Computational Case Studies}

\subsection{Method Implementation}

The present methodology has been implemented in the commercial package ANSYS Fluent facilitating the user defined functions (UDF). The implementation is based on a loosely coupled form. The baseline solvers of the system are taken to be a standard standalone fluid solver in a steady RANS, URANS or the scale adaptive simulation (SAS) mode, and a simple stand-alone steady conduction solver for the solid domain. As such, all temporal variations in the large (solid) time scale are given by the source terms for both 
fluid and solid domains (i.e. $\left.\left(\frac{\partial \tilde{U}}{\partial t}\right)_{M},\left(\frac{\partial \tilde{T}}{\partial t}\right)_{M}\right)$. On the other hand, the temporal terms for the short (fluid) time scale (i.e. $\left(\frac{\partial U}{\partial t}\right)_{m}$ ) will be activated for the URANS and SAS options. The compressible flow equations are deemed necessary for heat transfer problems of present interest and are solved by the pressure-based coupled method in Fluent.

The main solution iterations involved at a solid time step $\mathrm{M}$ as part of the overall loosely coupled CHT procedure are depicted in Fig.2.

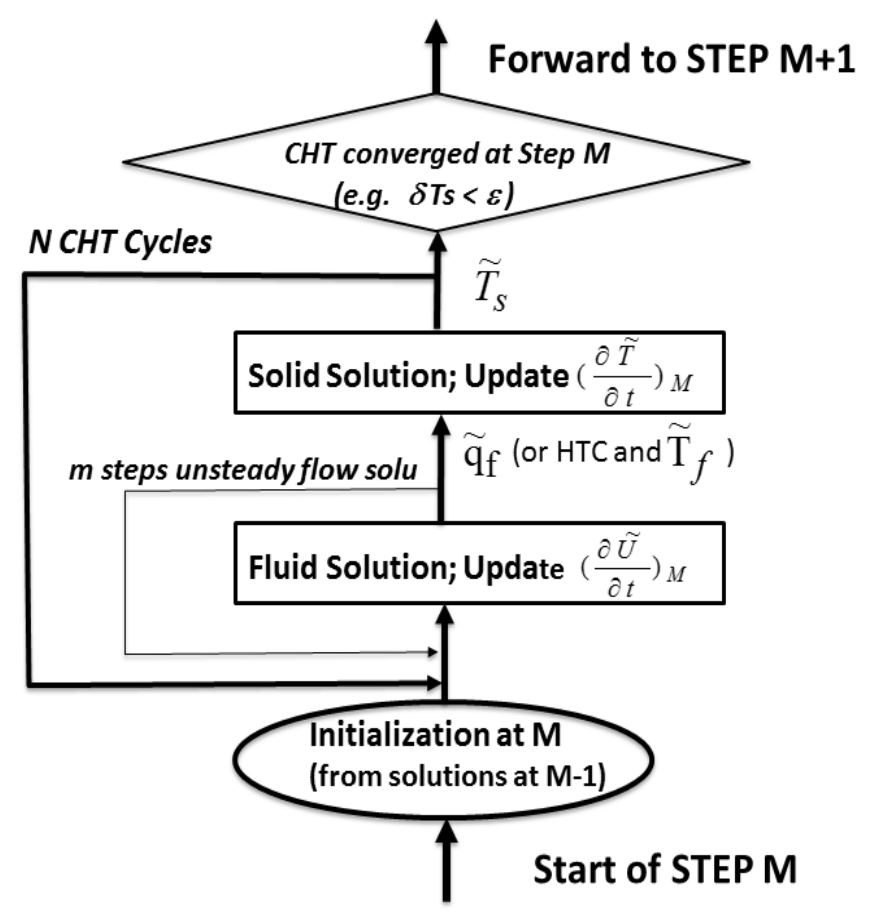

Fig.2, Iterative processes in a loosely coupled unsteady CHT procedure.

The actual process of iterating and exchanging data between the fluid and solid domains solver has been achieved using a MATLAB script. The sequence of the loosely coupled procedure can be described as:

- At the initial time $t=t_{o}$, a certain temperature distribution is imposed at solid wall, $\mathrm{T}_{\mathrm{s}}$ (as a Dirichlet boundary condition) in the fluid domain.

- Fluid domain solution is performed, to extract the wall heat flux $\mathrm{q}_{\mathrm{f}}$.

- In the case to calculate HTC, another fluid solution is obtained for a perturbed wall temperature $\mathrm{T}_{\mathrm{s}}+\Delta \mathrm{T}_{\mathrm{s}}$ where $\Delta \mathrm{T}_{\mathrm{s}}$ is small (typically $\Delta \mathrm{T}_{\mathrm{s}} / \mathrm{Ts}<5 \%$ to keep a local linearity). 
- The known heat flux, $\mathrm{q}_{\mathrm{f}}$ from the flow solution is transferred to the solid domain as a Neumann boundary condition.

- In the case for an imposed HTC $\mathrm{h}$ to the solid interface, the MATLAB routine is executed for the fluid driving temperature $\mathrm{T}_{\mathrm{f}}$

$$
T_{f}^{n}=T_{s}^{n}+\frac{q_{f}^{n}}{h}
$$

- With the imposed wall heat flux $\mathrm{q}_{\mathrm{f}}$ (or HTC and $\mathrm{T}_{\mathrm{f}}$ ), the solid conduction solution is obtained, leading to a temperature distribution in the solid.

- Then the solid surface temperature $T_{\mathrm{s}}$ is transferred to the fluid solver as a Dirichelt boundary condition again for the next coupling cycle.

The sequence above applies to both steady and transient CHT cases. The key difference in a transient CHT case is the updating of the unsteady source terms (i.e. $\left(\frac{\partial \tilde{U}}{\partial t}\right)_{M},\left(\frac{\partial \tilde{T}}{\partial t}\right)_{M}$ ) through the UDF facility. This is done after each step of $m$ sub-iterations for the fluid part, and after each cycle of $\mathrm{N}$ coupling cycles for the solid part respectively (Fig.2).

A basic flow solver verification for the convective heat transfer predictive capability is conducted for a flat plate boundary layer at a very low speed (free stream Mach number $=0.005)$, and a near adiabatic condition $\left(\mathrm{T}_{\mathrm{w}} / \mathrm{T}_{01}=0.95\right)$. The flow is laminar with $\operatorname{Re}=6.8 \times 10^{4}$. The computational results are compared with the corresponding analytical solutions as shown in Fig. 3.

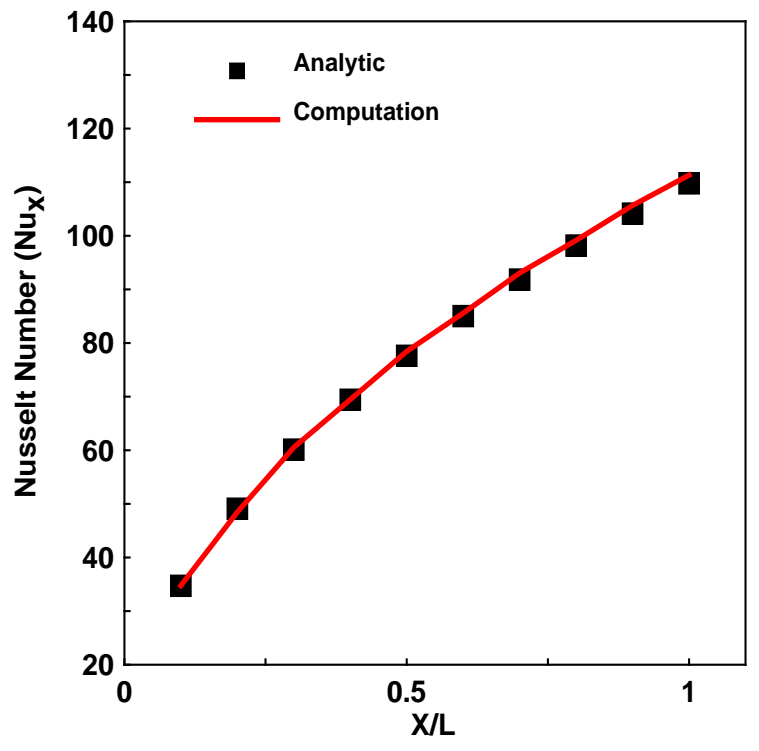

(a) Nusselt Number

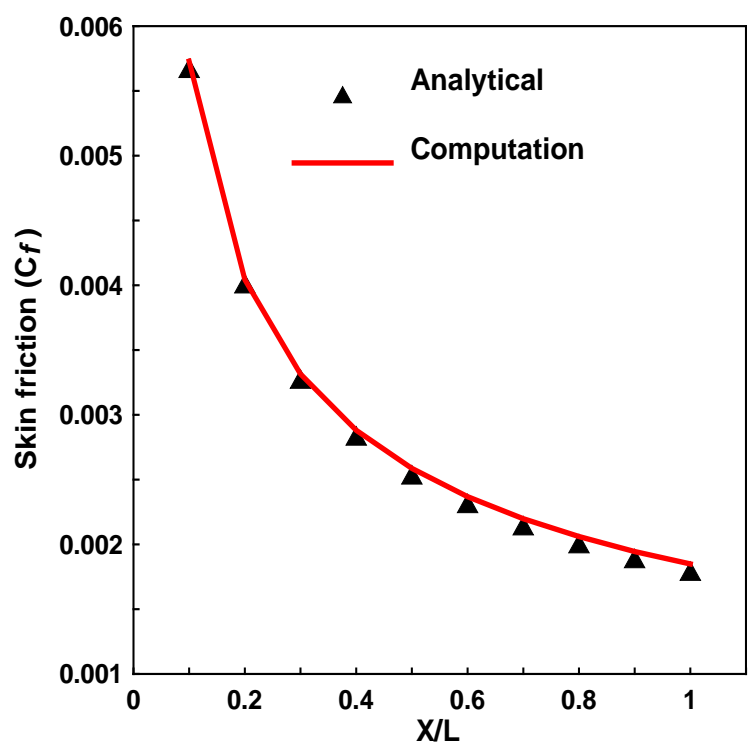

(b) Friction Coefficient

Fig.3, Basic Flow Solver Verification for Flat Plate Laminar Boundary Layer. 


\subsection{Steady CHT for Simple Duct}

The first conjugate heat transfer test case is for a simple 2D duct configuration as shown in Fig.4. The fluid domain is of length $\mathrm{L}=1 \mathrm{~m}$, height $0.1 \mathrm{~m}$. The top boundary of the fluid domain is an adiabatic wall. The solid domain is of height $0.2 \mathrm{~m}$, and its top boundary is the interface with the fluid domain. The two side boundaries and the bottom boundary of the solid domain are subject to a convective boundary condition with a constant HTC $\left(\mathrm{h}=100 \mathrm{~W} / \mathrm{m}^{2} \mathrm{~K}^{-1}\right)$ and a constant environment fluid temperature $\left(\mathrm{T}_{\mathrm{f}}=\right.$ $300 \mathrm{~K})$. Steel is taken as the solid material with a thermal conductivity of $16.3 \mathrm{~W} / \mathrm{m} . \mathrm{K}^{-1}$.

The fluid domain is subject to an inflow at $10 \mathrm{~m} / \mathrm{s}$ with $10 \%$ turbulence intensity. The Reynolds number based on the duct height is $2.28 \times 10^{5}$. The RANS solver is used for the fluid domain with the shear stress transport (SST) model for turbulence closure. The fluid domain is meshed with $100 \times 500$ mesh points, and a $50 \times 500$ mesh is used for the solid domain. On the fluid side of the interface, $y+$ for the $1^{\text {st }}$ mesh cell distance from the wall is kept below 10, so that the non-slip wall condition for the fluid side of the boundary is applied.

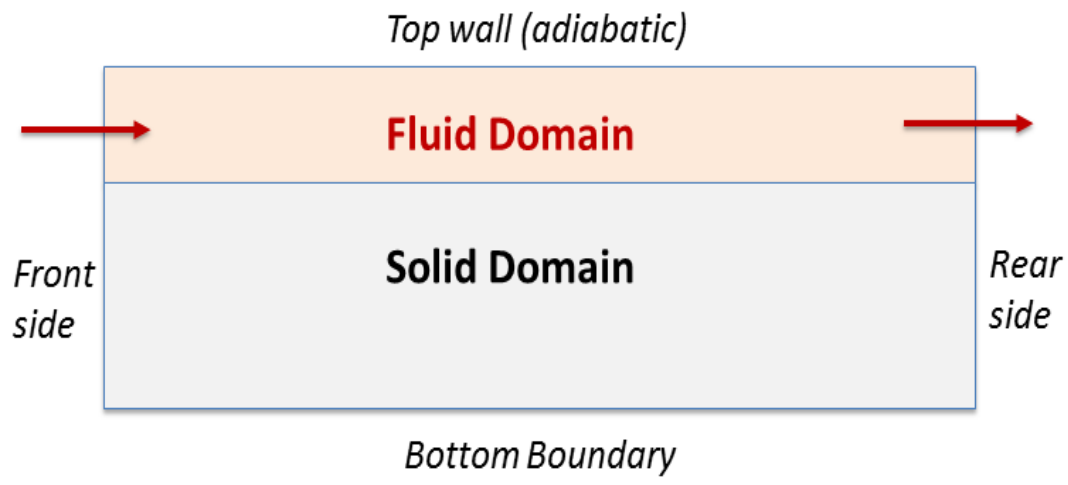

Fig.4, Fluid-solid domains for simple duct configuration.

The present loosely coupled CHT solution is compared to the direct fully coupled CHT solution of Fluent. Figure 5 shows dimensionless interface wall temperatures, compared to those calculated by the direct fully coupled CHT of Fluent. It can be seen that the loosely coupled and the direct fully coupled CHT solutions are in excellent agreement. Figure 6 shows the wall heat fluxes normalised by $\mathrm{q}_{\mathrm{ref}}\left(\mathrm{q}_{\mathrm{ref}}=\mathrm{k}_{01}\left(\mathrm{~T}_{01}-\mathrm{Tw}\right) / \mathrm{L}\right.$, where $\mathrm{k}_{01}$ is the fluid thermal conductivity at the inflow stagnation condition) on both the solid and fluid sides of the interface. It is clear that the predicted heat fluxes on both 
sides of the interface match each other very well, a clear manifestation of the physical interface condition.

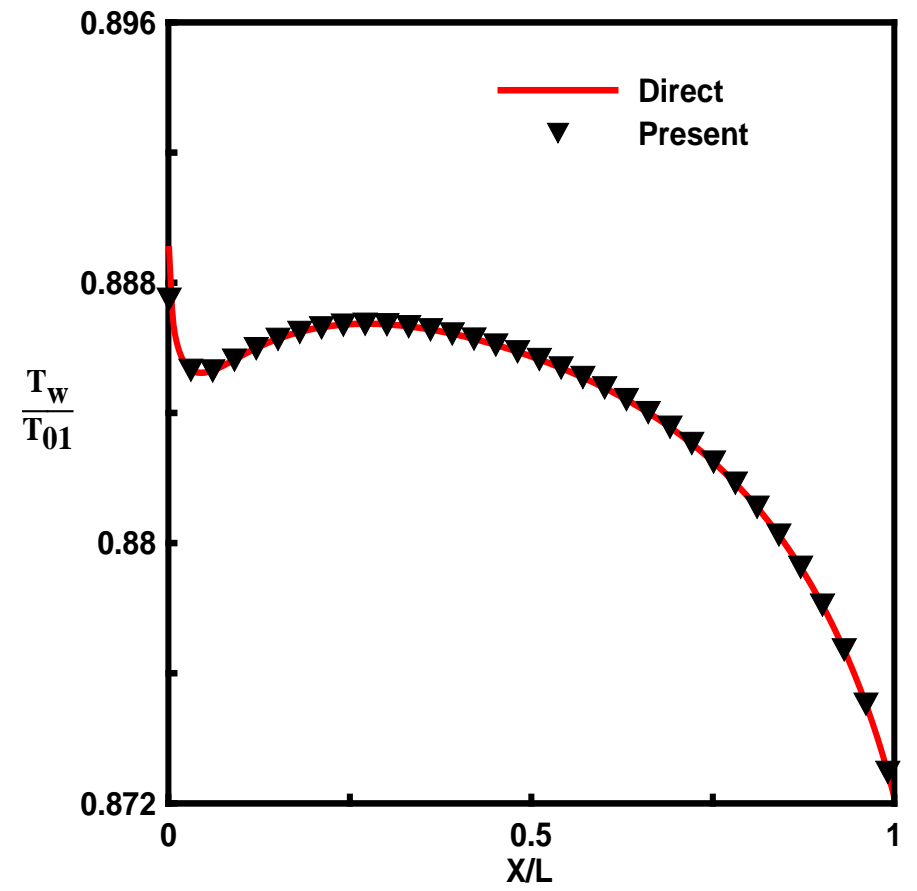

Fig.5, Comparison of fluid-solid interface temperatures between the present loosely coupled ('Present') and the direct fully coupled CHT ('Direct') solutions.

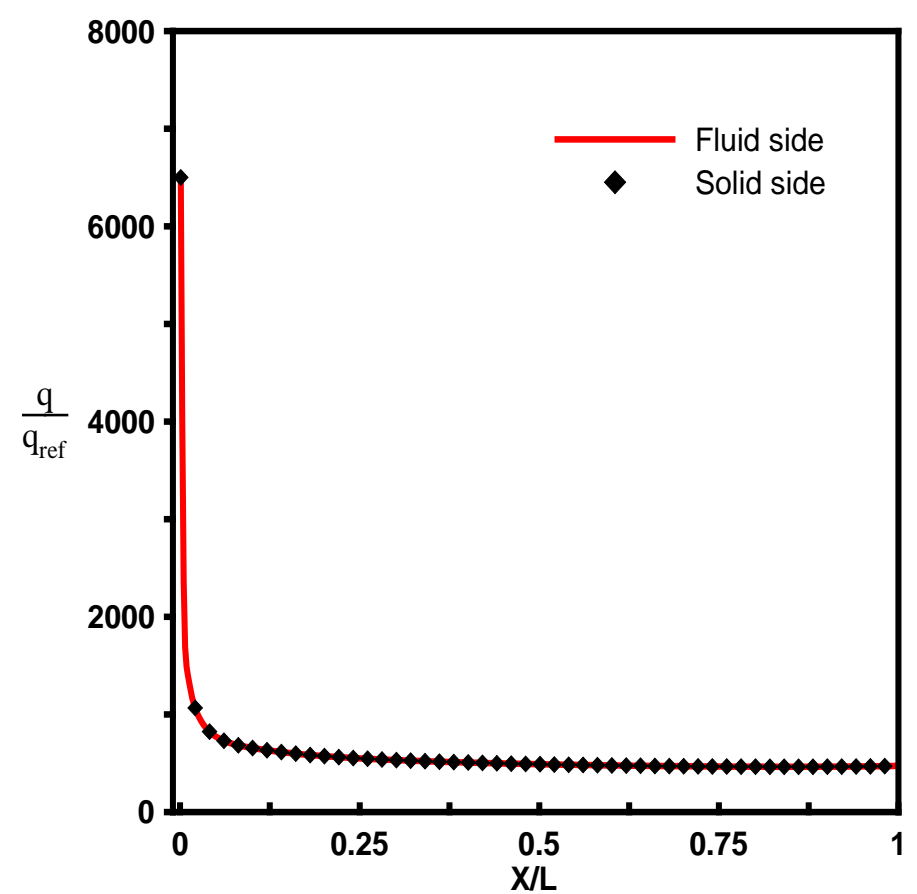

Fig. 6, Heat fluxes on both sides of fluid-solid interface. 


\subsection{Influence of Interface Methods on CHT Coupling Convergence}

In a loosely coupled CHT, the iterations between setting the fluid wall temperature from a solid solution and setting the solid wall heat flux from a fluid solution tend to have slower convergence rate compared to the HTC based interfacing method. We however would prefer to make use of the $\mathrm{T}_{\mathrm{S}}-\mathrm{q}_{\mathrm{f}}$ method even just partially, as it presents the most direct manifestation of the physical $\mathrm{T}$ and $\mathrm{q}$ continuity conditions once the interfacing iterations converge. A new hybrid method to combine these two is thus adopted to consolidate the conflicting characteristics between the convergence speed and accuracy.

Here we consider the computational time costs with respect to the convergence of the CHT coupling in terms of changes in wall temperatures between successive coupling cycles. The convergence histories of the normalised wall temperature error-norm (in log scale) are given in Fig.7. As most computational costs are due to the fluid solution, the computational costs for all these methods are normalised by that of a stand-alone fluid solution. The number of cycles required for each interface method is also indicated by the number of symbol points plotted. Thus, the direct $\mathrm{T}_{\mathrm{S}}-\mathrm{q}_{\mathrm{f}}$ interfacing treatment needs 7 iterations to converge, compared to 3 cycles required by the HTC based method. Worth noting is that the convergence rate (indicated by the slope of the curves) of the HTC based treatment is significantly higher in its initial cycle than that in the final cycle.

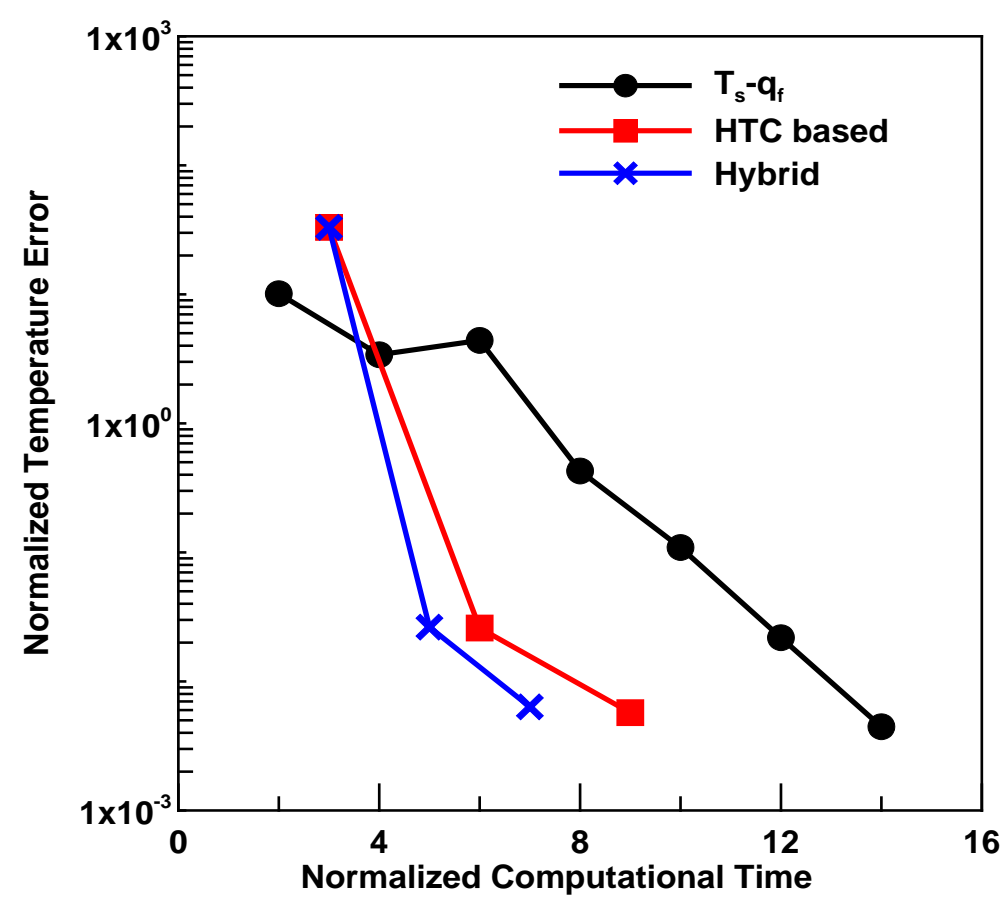

Fig. 7, Comparison of normalised computational time costs 
The hybrid method first executes 1 cycle using the HTC based method followed by 2 cycles of the Ts- $\mathrm{q}_{\mathrm{f}}$ treatment. It can be seen that a similar convergence rate to that of the HTC based can be achieved. A further gain in computational efficiency by the hybrid method is achieved due to the fact that HTC based method needs two fluid solutions for each cycle, compared to only 1 fluid solution needed for a $T_{s}-q_{f}$ cycle. A very useful feature of the hybrid implementation is thus observed that it takes the advantage of the HTC based (Robin-like) method in first driving down the near wall temperature errors in the solid domain very effectively before enforcing the direct physical conditions by the $\mathrm{T}_{\mathrm{S}}-\mathrm{q}_{\mathrm{f}}$ treatment as desired.

\subsection{Transient CHT for Duct Flow with Varying Inlet Conditions}

Now we examine the transient conjugate heat transfer capability of the present loosely coupled CHT implementation. We first look at a slow transient case where the quasi-steady assumption for the fluid part should be perfectly valid. Then we will examine the unsteady effect on the fluid part under a fast ramping condition.

The same duct configuration as in the previous example is used. The inflow however is now subject to linear variations in fluid velocity $(3 \mathrm{~m} / \mathrm{s}-8 \mathrm{~m} / \mathrm{s})$ and temperature $(350 \mathrm{~K}-$ $450 \mathrm{~K})$ in 5 minutes. The overall time scale for these simple linear variations is very long so that the flow response in time can be considered to be completely quasi-steady. The loosely coupled CHT procedure is marched in time at a physical time step of 1 minute.

Figure 8 shows the instantaneous interface wall temperatures at three time instants by the present loosely coupled CHT method, compared with those by the direct CHT method. We can readily observe the very good agreement between the two solutions. 


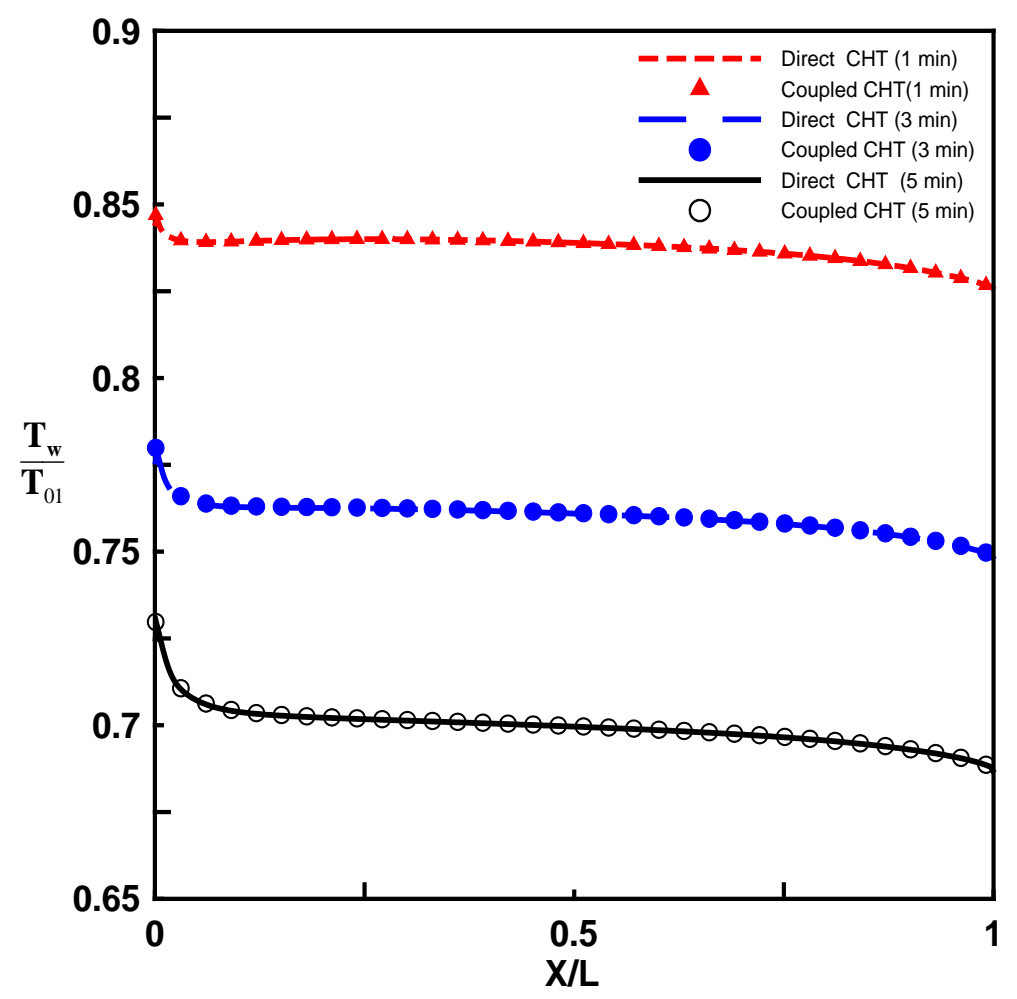

Fig.8, Instantaneous Wall Temperature Distributions at Different Time Instants.

Having validated the basic unsteady CHT capability of the methodology and implementation, we now turn the attention to a fast ramping condition where the unsteady effect on the fluid part may not be negligible. The inlet velocity and temperature are now linearly ramped up from $10 \mathrm{~m} / \mathrm{s}$ to $40 \mathrm{~m} / \mathrm{s}$, and from $350 \mathrm{~K}$ to $650 \mathrm{~K}$ respectively in 0.1 second. A physical time step of $0.01 \mathrm{~s}$ is taken in this case.

The results are compared between the quasi-steady fluid model (without the temporal fluid source term) and the unsteady fluid model (with the temporal fluid source term). The non-dimensional heat fluxes and wall temperatures on the fluid-solid interface are shown in Fig.9. Also plotted are those from the direct fully coupled CHT as the reference solution. It is clear that the present loosely coupled CHT solution is in excellent agreement with the direct solution. However, a significant over-prediction (up to $100 \%$ ) of heat transfer is noticed in the results by the quasi steady fluid model.

Although as the time passes by, the errors become smaller (Fig.9b), the impact of the temporal errors associated with the quasi-steady model however might not be negligible. This is because the transient load in the solid domain at a later time will depend on the total integrated heat transferred across all the boundaries during the time period prior to that instant. So care should be taken in adopting a quasi-steady fluid model for predicting transient thermal loads, even for some rapid ramping in a very short 
period of time of an otherwise seemingly slow process. Furthermore it is pointed out that the present method including the unsteady fluid source terms should consume largely the same amount of computing time as the previous quasi-steady transient approach without the unsteady fluid source term. This contrast underlines the clear advantage of the present method with a much expanded validity at little or no extra cost.
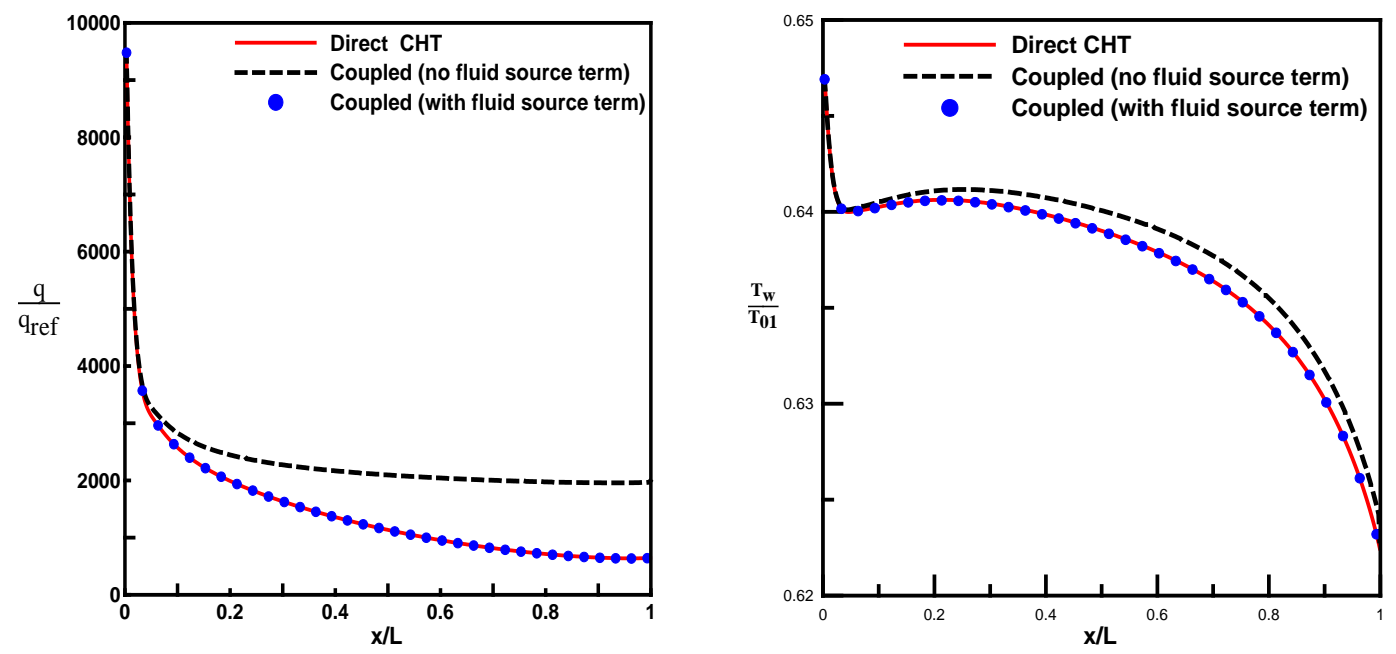

(a) $\mathrm{t}=\mathbf{0 . 0 5 \mathrm { s }}$
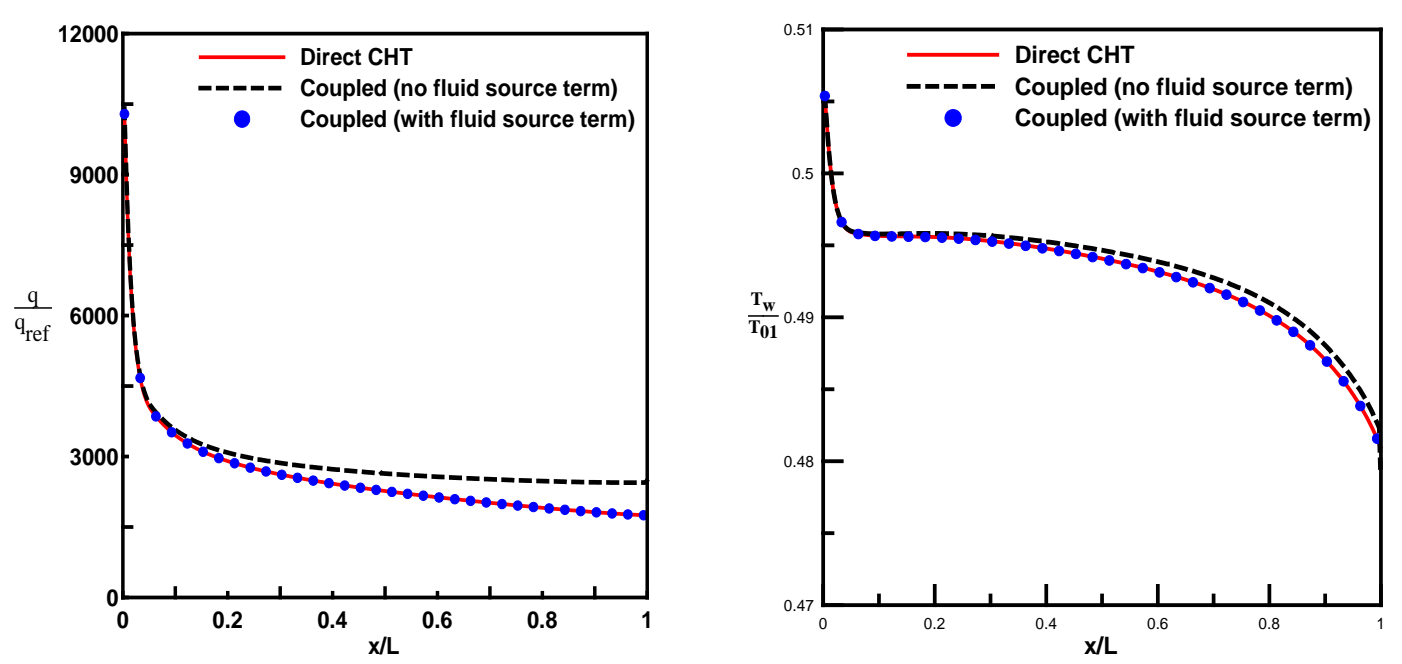

(b) $\mathrm{t}=0.1 \mathrm{~s}$

Fig.9, Instantaneous heat fluxes and temperatures on interface at two time instants.

\subsection{Transient CHT for Duct Flow with Self-Excited Unsteadiness}

In the transient CHT case presented in the previous section, the quasi-steady fluid model assumption is challenged by the transient CHT behaviour in response to fast changing inflow boundary conditions. For that case, the present method is shown to be of the same accuracy as the direct fully coupled solution. The advantage of the present 
method over the direct method can be more clearly shown for cases where the prevailing fluid time scale is much smaller due to the requirement to resolve fast and dominant unsteady fluid mechanisms. In this context, self-excited fluid instabilities (e.g. vortex shedding or more commonly resolved turbulence eddies) are examples of common interest. In these situations, the direct fully coupled CHT solutions can be very timeconsuming.

In this present test case, a fluid-solid configuration with prevailing unsteady fluid dynamic structures of small time scales is taken, as shown in Fig.10. The main source of fast unsteadiness comes from the vortex shedding from a partially blocked inlet, relevant to part-load conditions during a start-up or shut-down process. The fluid domain is bounded by the left side wall and the bottom wall, both of which are treated as an adiabatic boundary. The solid domain is bounded by the top side and right side boundaries. Both are subject to a convective boundary condition with a HTC of 100 $\mathrm{W} / \mathrm{m}^{2} \mathrm{~K}$, and an environmental fluid temperature of $300 \mathrm{~K}$. The fluid-solid interface consists of a vertical and a horizontal part.

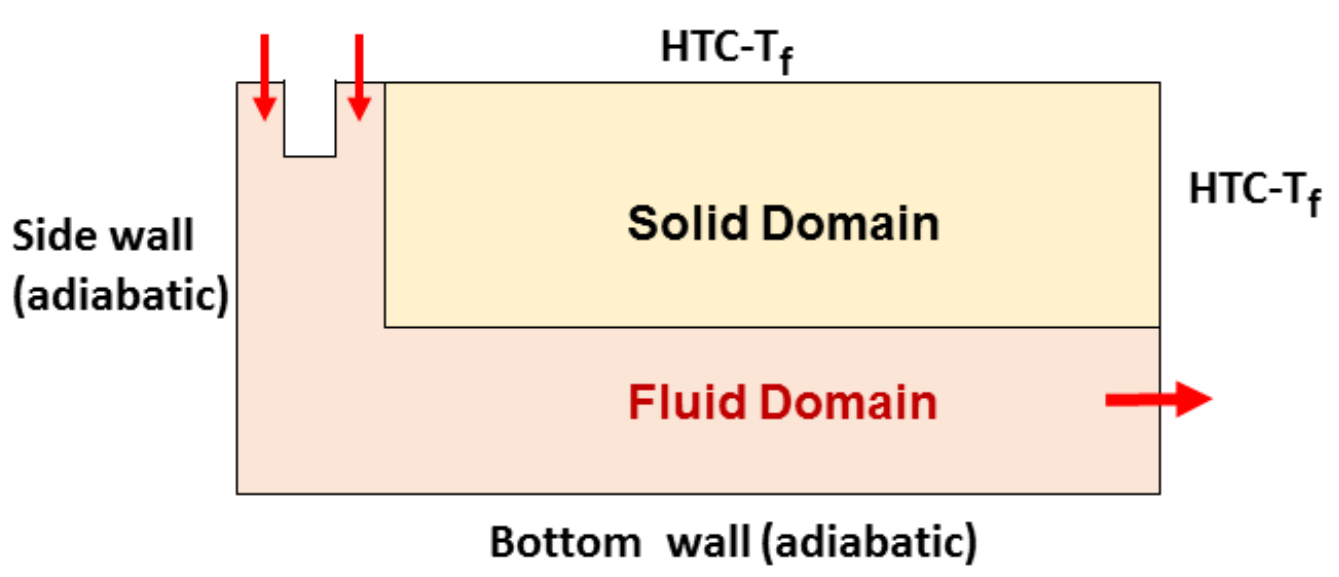

Fig.10, Computational domain and boundary conditions.

The inflow condition at the two open slots of the partially blocked inlet has a flow velocity of $10 \mathrm{~m} / \mathrm{s}$, a stagnation temperature of 350K. The Reynolds number based the inflow velocity and the length of the horizontal part of the duct is $3.6 \times 10^{5}$. The $2 \mathrm{D}$ computational domain has a total of 254,000 mesh cells (130,000 mesh cells in the fluid domain and 124,000 mesh cells in the solid domain). Self-excited unsteady disturbances originated from both the partially blocked inlet and the sharp turn from the vertical part 
to the horizontal one of the duct are further complicated by their interactions, thus leading to a strong dependence of the CHT solution on the flow modelling fidelity.

Fig.11 shows the instantaneous snapshots of vortical flow patterns computed by three flow models: steady RANS, unsteady RANS, and the scale adaptive simulation (SAS). The SAS solution clearly picks up smaller vortices than does the URANS, indicating the preliminary eddy resolving capability of the SAS model. The turbulence eddy resolving capability of the SAS towards LES is also illustrated in the time history comparison between the URANS and the SAS models for a mesh point at the middle of the duct as shown in Fig.12.
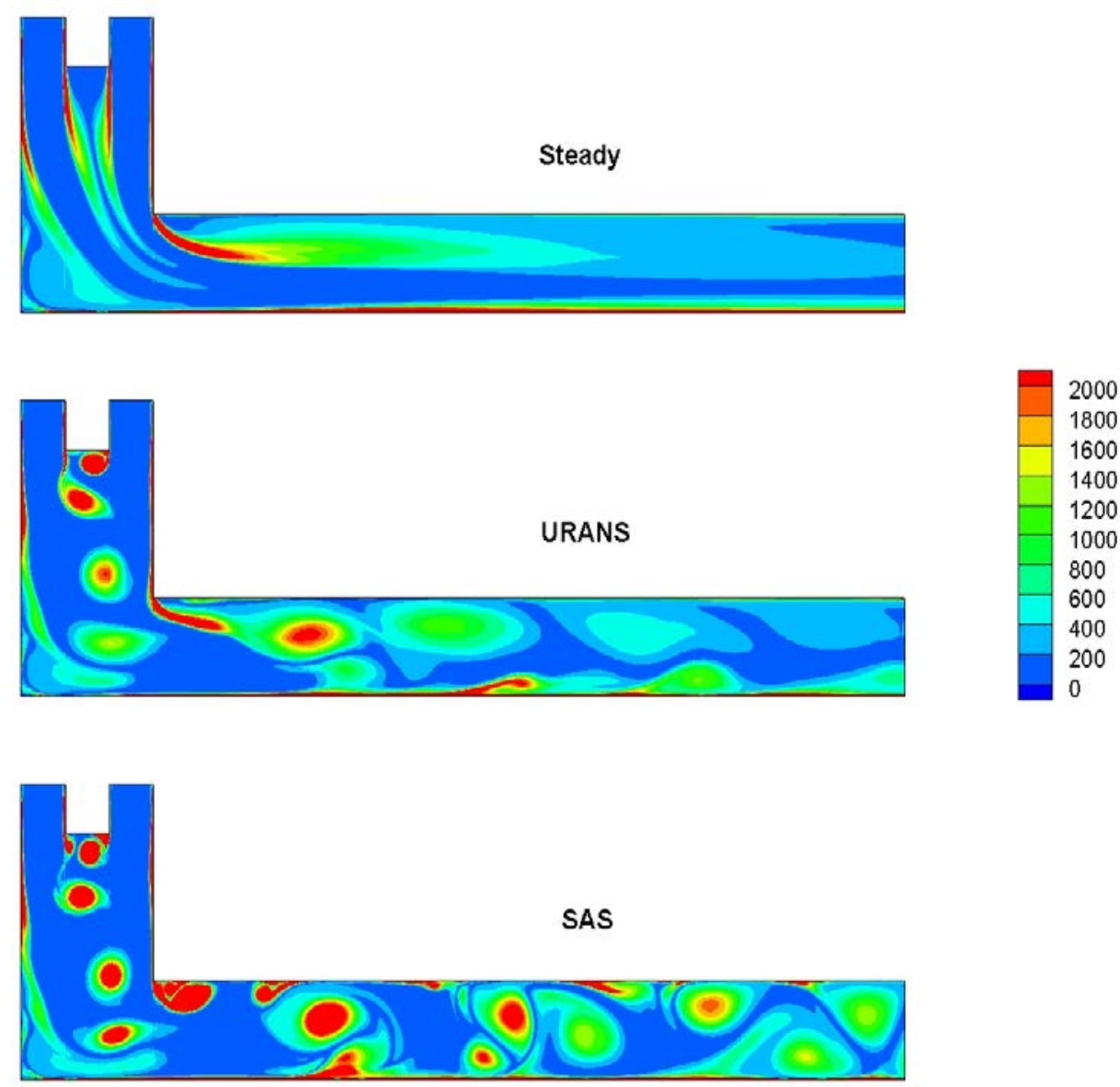

Fig 11, Instantaneous Contours of Vorticity Magnitude

The steady flow solution (top of Fig.11) failing to capture any unsteady vortical flow activities as expected would pose neither restriction nor requirement on the temporal resolution. However for both the URANS and the SAS solutions, the temporal 
resolution is clearly a relevant issue which poses a requirement on the suitable time step size, even just from a viewpoint of numerical consistency.

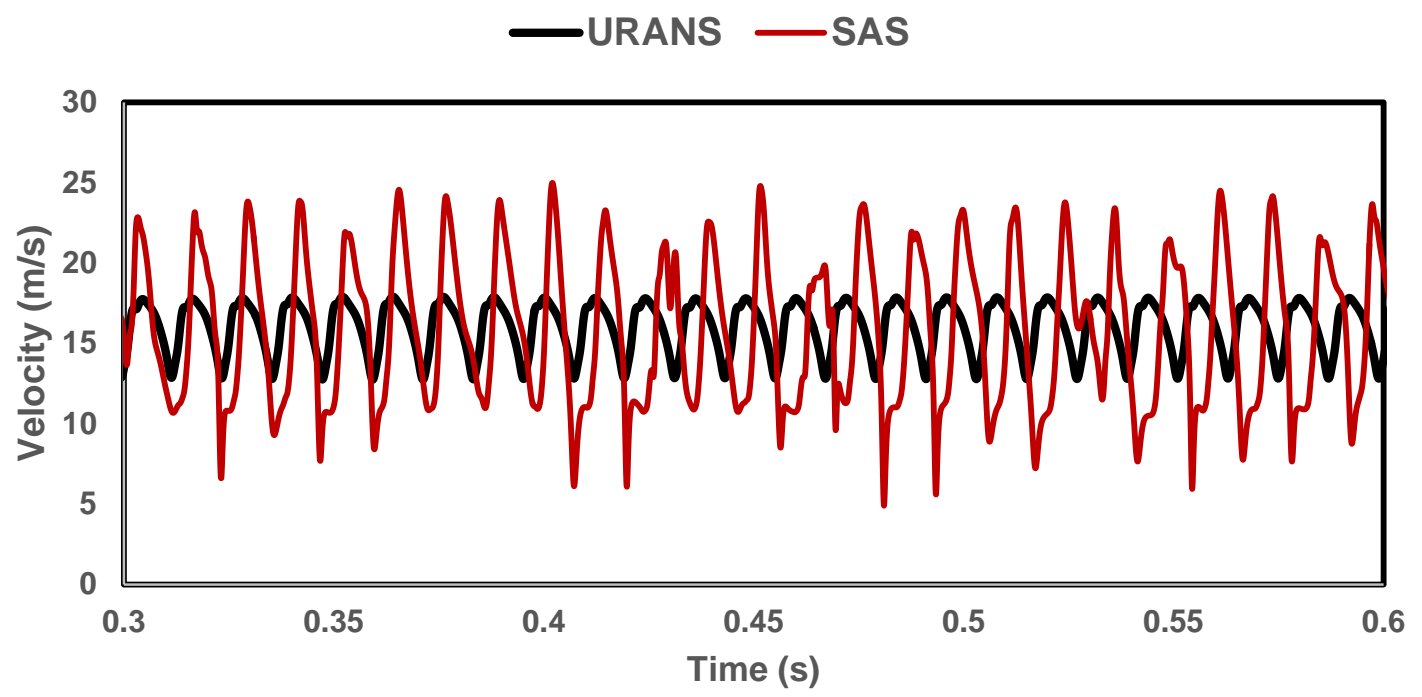

Fig.12, Velocity time histories at the mid-duct point.

It is recognised that a SAS solution (or a LES solution in general) can be dependent on the time step size, and examining the time-step dependence of SAS is beyond the scope of the present work. However, for modelling consistence we should consider the temporal resolution requirement of the URANS, and thus its implication for the computational efficiency of the present method in relation to the direct solution. Given this consideration, the time step dependence is examined for the URANS, in terms of the time-averaged heat transfer on the solid wall surface. The results with three different time step sizes are shown in Fig.13. For this case, it is found that a time step independent URANS solution can be obtained when the physical time step is equal to or smaller than 0.0001 s (i.e. $100 \mu$ s).

Based on this temporal resolution requirement, the physical time step is taken to be $100 \mu$ s to proceed with a transient CHT coupling. The inflow velocity is changed from $5 \mathrm{~m} / \mathrm{s}$ to $10 \mathrm{~m} / \mathrm{s}$ and the inlet temperature is changed from $300 \mathrm{~K}$ to $400 \mathrm{~K}$ in 60 seconds. The present URANS CHT solution is compared to the corresponding steady RANS solution in terms of the interface temperatures (Fig.14) and heat fluxes (Fig.15). Also shown are the corresponding results using the SAS model. We can clearly see the impact of the flow modelling fidelities. The difference in the heat flux between the unsteady flow models and the steady one can be up to $50 \%$. 


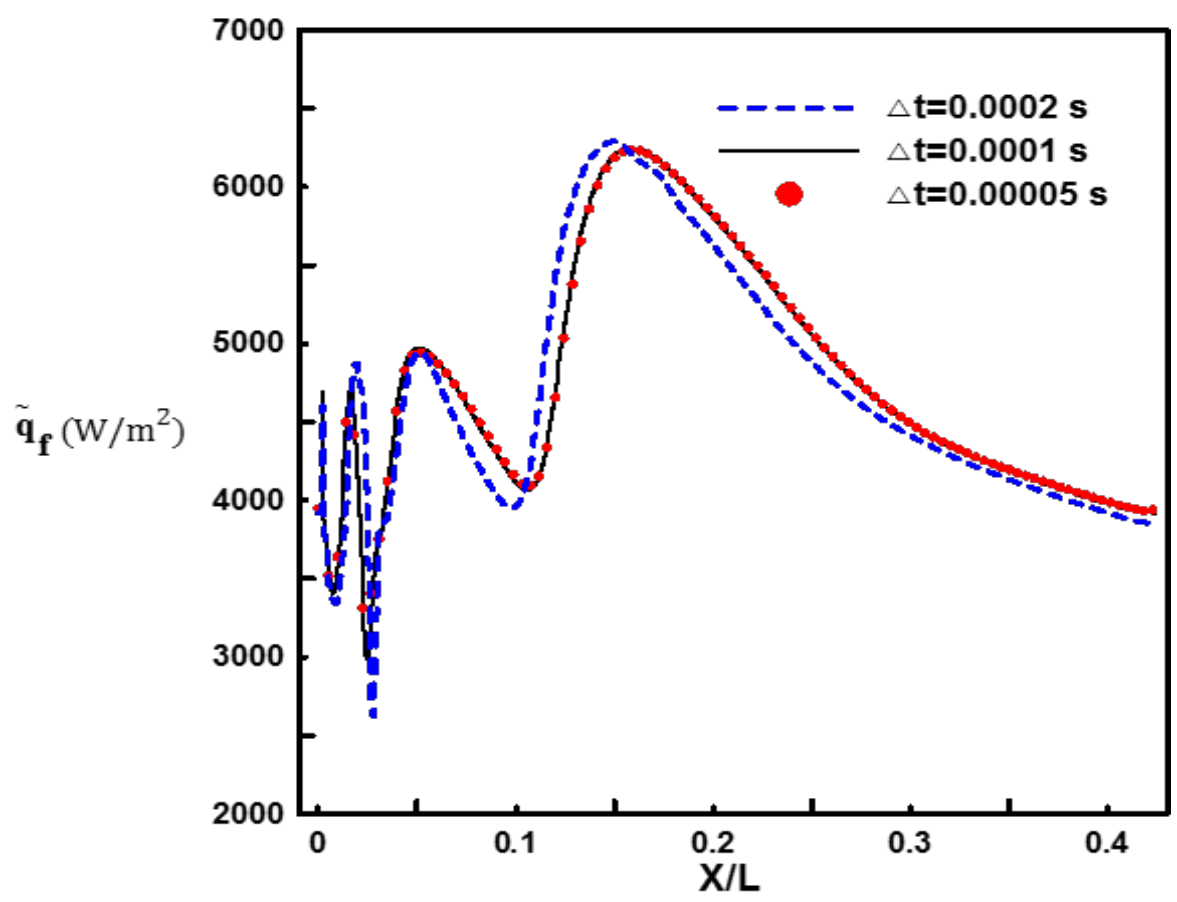

Fig.13, Time averaged heat flux distributions with different time-steps for URANS (distance is measured from the start of the horizontal part of the duct).

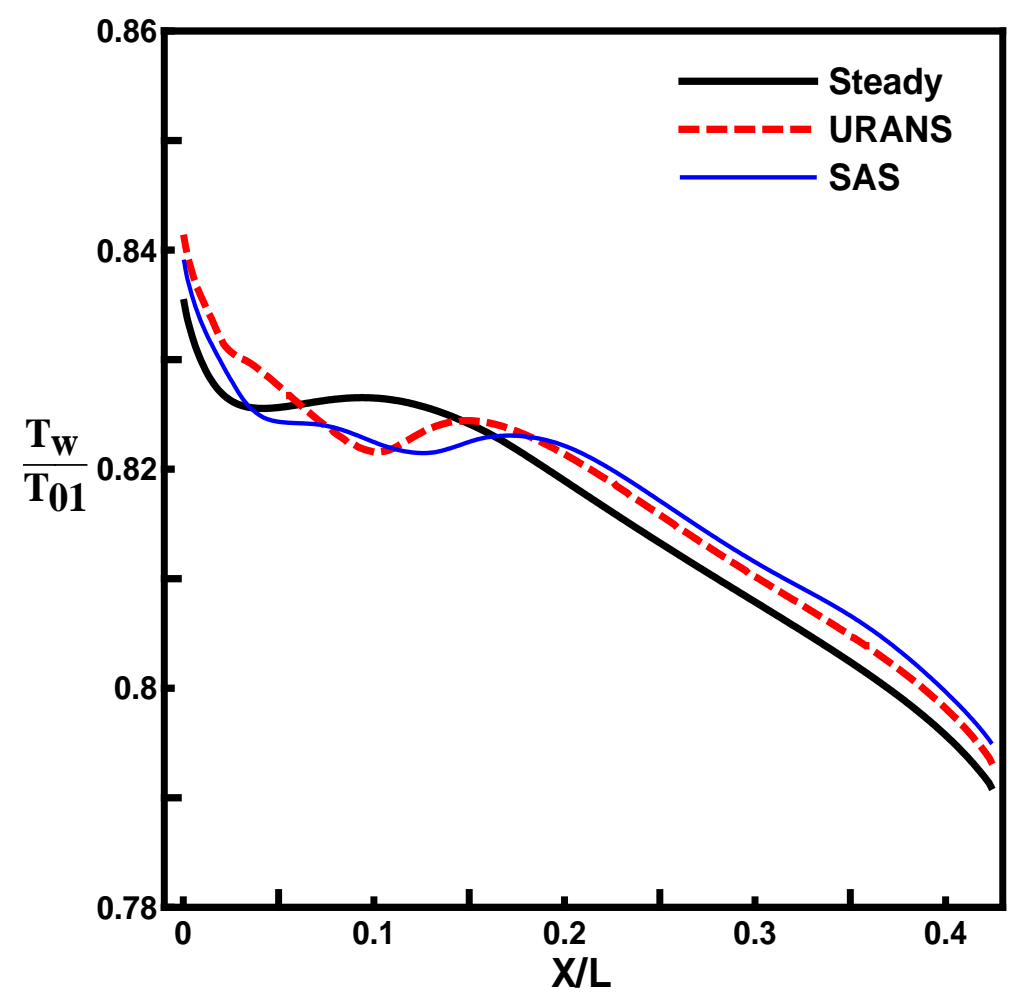

Fig.14, Instantaneous temperatures on the fluid-solid interface (at time $=60 \mathrm{~s}$ ) 


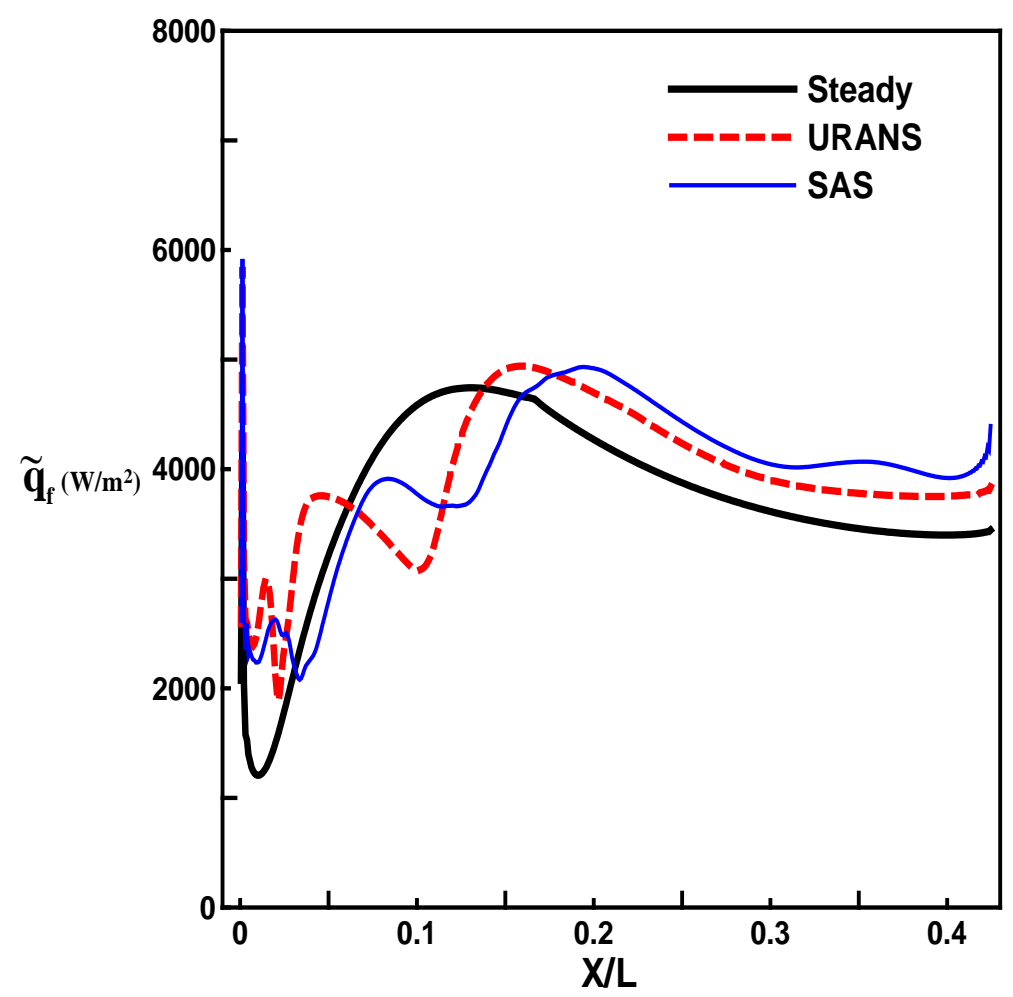

Fig.15, Instantaneous heat fluxes on the fluid-solid interface (at time=60 s).

Finally we make some comments regarding the computational costs. The computational efficiency of the present multi-scale integration would depend on the length of time period (thus the number of time steps) required for getting adequately time averaged parameters for each CHT coupling cycle. In the present test case, time averaging either $\mathrm{q}$ or $\mathrm{T}$ is carried in a simple moving averaging form, shown to be effective for nondeterministic unsteady disturbances [23]:

$\tilde{q}^{m}=\frac{m-1}{m} \tilde{q}^{m-1}+\frac{1}{m} q$

In the present case, 9000 time steps at a step size at $100 \mu$ s are used for each fluid solution, so that 5 cycles of the CHT coupling require 45,000 steps. On the other hand, if a direct solution is adopted, 600,000 time steps will be needed to march through 60 seconds. Thus, a factor of 13 in computational speed gain is obtained by using the present multi-scale integration method for this particular transient CHT test case. The computational gain can be further enhanced if a short time than $0.9 \mathrm{~s}$ is taken for the local time averaging. As indicated by Fig.13, 0.9 second appears to be much more than adequately required for the local time-averaging.

A general indication of the potential computational gain by using the multi-scale approach may be considered for a case where a high fidelity LES solution is adopted for 
the fluid domain. In this case a typical time step required for temporal resolution of fluid side can be easily as small as in microns. If on the other hand, the time step required for predicting transient temperature field in the solid domain is in seconds, we should then have a huge time step disparity from the temporal resolution requirements, i.e. $\Delta \mathrm{t}_{\mathrm{s}} / \Delta \mathrm{t}_{\mathrm{f}}$ $\sim 10^{6}$. This would make a directly coupled CHT solution prohibitively time consuming as it will have to use a very small fluid time step, thus a huge number of steps to cover the large solid time step. Now if we use the loosely coupled multi-scale approach, the time-scale to be covered in the local time integration only needs to be long enough for obtaining adequately time averaged parameters during each coupling cycle at each solid time step. In this case, if we can complete the time integration in the fluid domain in $10^{3}-10^{4}$ steps as illustrated $n$ the present test case, it would lead to a computational speed gain by a factor of $10^{2}-10^{3}$. This appealing aspect should be further examined in computational case studies and application tests in future work.

\section{Concluding Remarks}

Existing transient conjugate heat transfer (CHT) methods adopt a quasi-steady assumption for the fluid domain, and there is thus a lack of CHT methods applicable for problems of a wide range of time-scales. In the present paper, a general framework is presented based on a multi-scale methodology. A scale-dependent flow variable decomposition is adopted to split long time scales typically associated with a solid domain and much smaller fluid time scales. A corresponding time-step split is also adopted. Consequently, the fluid equations can be integrated on the two time scales very effectively. The resultant fluid-solid coupled conjugate system equations formulated in a triple-timing form can be solved efficiently. In addition, a simple new hybrid fluid-solid interface method is adopted to achieve a good convergence whist maintaining the direct physical conditions of temperature and heat flux continuity across the interface. The methodology has been implemented in a loosely coupled procedure in a common commercial code for validations and demonstrations. The computational results including different time scale characteristics demonstrate the validity, correctness of the implementation and a wider applicability of the present methodology. 


\section{Acknowledgement}

The project is funded by EPSRC with a partial financial support from GE-ALSTOM Power, which is gratefully acknowledged.

\section{References}

[1] Moffat, R. J., What's New in Convective Heat Transfer? International Journal of Heat and Fluid Flow, 1998, Vol.19, pp90-101.

[2] Maffulli, R., and He, L., Wall Temperature Effects on Heat Transfer Coefficient for High Pressure Turbines, AIAA Journal of Propulsion and Power, 2014, Vol.30, No. 4, pp.1080-1090, July 2014.

[3] He, L., and Oldfield, MLG. Unsteady Conjugate Heat Transfer modelling, Journal of Turbomachinery, 2011, Vo1.133, No.3, 2011.

[4] Sun, Z, Chew JW, Hills, N, Volkov K, and Barnes C. Efficient Finite Element Analysis/Computational Fluid Dynamics Thermal Coupling for Engineering Applications, Journal of Turbomachinery, 2010, Vo1.132, No.3.

[5] Ganine, V, Javiya, U, Hills, N, and Chew JW, Coupled Fluid-Structure Transient Thermal Analysis of a Gas Turbine Internal Air System with Multiple Cavities, J of Engineering for Gas Turbines and Power, 2012, Vol.135, No.10.

[6] Marinescu, G, Stein, P and Sell M, Natural Cooling and Startup of Steam Turbines: Validity of the Over-Conductivity Function, $J$ of Engineering for Gas Turbine and Power, 2015, Vol.137, No.11.

[7] He, L. Fourier Spectral Method for Multi-scale Aerothermal Analysis, International Journal of Computational Fluid Dynamics, 2013, Vol.27, No2, pp118-129.

[8] Shahi, M., Kok, J.B.W., Casado, J.C R. and Pozarlik, A.K. Study of unsteady heat transfer as a key parameter to characterize limit cycle of high amplitude pressure oscillations. ASME Paper GT2014-26311, 2014.

[9] Radenac E, Gressier J, Millan P. Methodology of Numerical Coupling for Transient Conjugate Heat Transfer. Computers \& Fluids. 2014. Vol.100, pp 95-107. 
[10] Shen C, Sun F, and Xia X. Analysis on transient conjugate heat transfer in gapcavity-gap structure heated by high speed airflow. International Journal of Heat and Mass Transfer, 2013, Vol.67, pp.1030-1038.

[11] Errera M, and Turpin G. Temporal multiscale strategies for conjugate heat transfer problems, Journal of Coupled Systems and Multiscale Dynamics, 2013, Vol.1, pp 89-98.

[12] Wang Z, Corral, R, Caster J. and Pastor. G. Analysis and Improvement of a Loosely Coupled Fluid-Solid Heat Transfer Method, ASME Paper GT201394332, 2013.

[13] Errera M, and Baqué B. A quasi-dynamic procedure for coupled thermal simulations, International Journal for Numerical Methods in Fluids, 2013, Vol.72, pp1183-1206.

[14] Giles, M.B., Stability analysis of numerical interface conditions in fluidstructure thermal analysis, International Journal for Numerical Methods in Fluids, 1997, Vol.25, No.4, pp421-436.

[15] Khanal, B., He, L., Northall, J., and Adami, P., Analysis of radial migration of hot-streak in swirling flow through HP turbine stage, Journal of Turbomachinery, 2013, Vol.135, No.4.

[16] Schmidt M, Starke C., Coupled heat-transfer simulations of turbines in consideration of unsteady flows, International Journal of Thermal Sciences, 2015, Vol.96, pp305-318.

[17] Duchaine F, Corpron A, Pons L, Moureau V, Nicoud F, and Poinsot T., Development and assessment of a coupled strategy for conjugate heat transfer with Large Eddy Simulation: Application to a cooled turbine blade”. International Journal of Heat and Fluid Flow, 2009, Vol.30, pp:1129-1141.

[18] Duchaine, F, Boileau,,M., SommererY., and Poinsot T. , Large Eddy Simulation of Flow and Heat Transfer Around Two Square Cylinders in a Tandem Arrangement, Journal of Heat Transfer, 2014, Vol.136, No.10, 101702.

[19] Menter, FR, Kuntz, M and Bender R, A Scale-Adaptive Simulation Model for Turbulent Flow Predictions, AIAA Paper 2003-0767, 2003.

[20] Jameson, A., Time-Dependent Calculations using Multi-Grid, with Applications to Unsteady Flows past Airfoil and Wings, AIAA Paper 91-1596, 1991. 
[21] Batten, P., Goldberg, U., and Chakravarthy, S., Interfacing statistical turbulence closures with large-eddy simulation. AIAA Journal, 2004, Vol.42, No.3, pp485492.

[22] Perelman, T.L. On Conjugated Problems of Heat Transfer, International Journal of Heat and Mass Transfer, 1961, Vol.3. No.4, pp293-303.

[23] He, L., Harmonic Solution of Unsteady Flow around Blade with Separation, AIAA Journal, 2008, Vol.46, No.6. 